\title{
Cognitive-behavioural therapy $v$. social activity therapy for people with psychosis and a history of violence: randomised controlled trial
}

Gillian Haddock, Christine Barrowclough, Jennifer J. Shaw, Graham Dunn, Raymond W. Novaco and Nicholas Tarrier

\section{Background}

Aggression and violence are serious problems in schizophrenia. Cognitive-behavioural therapy (CBT) has been shown to be an effective treatment for psychosis although there have been no studies to date evaluating the impact of CBT for people with psychosis and a history of violence.

\section{Aims}

To investigate the effectiveness of CBT on violence, anger, psychosis and risk outcomes with people who had a diagnosis of schizophrenia and a history of violence.

\section{Method}

This was a single-blind randomised controlled trial of CBT $v$. social activity therapy (SAT) with a primary outcome of violence and secondary outcomes of anger, symptoms, functioning and risk. Outcomes were evaluated by masked assessors at 6 and 12 months (trial registration: NRR N050087441).

\section{Results}

Significant benefits were shown for CBT compared with control over the intervention and follow-up period on violence, delusions and risk management.

\section{Conclusions}

Cognitive-behavioural therapy targeted at psychosis and anger may be an effective treatment for reducing the occurrence of violence and further investigation of its benefits is warranted.

\section{Declaration of interest}

None. Funding detailed in Acknowledgements.
There is significant public concern about violence and aggression in people with schizophrenia. Furthermore, violence in schizophrenia can present considerable challenges to mental health services and take up a large proportion of staff time and resources. Little is known about how to reduce such violence and aggression. Meta-analyses have shown that cognitivebehavioural therapy (CBT) significantly reduces psychotic symptoms in schizophrenia. ${ }^{1,2}$ However, the majority of trials have been carried out with people with chronic, treatmentresistant symptoms living in the community. There has been little evaluation of CBT with people with complex presentations such as those who are aggressive or violent. ${ }^{3}$ Violence and aggression appear to be associated with the presence of delusional symptoms and anger. ${ }^{4,5}$ Anger has been successfully treated using $\mathrm{CBT}^{6}$ suggesting that CBT for psychosis could be modified to specifically reduce anger and aggression as well as psychotic symptoms. Research is needed to evaluate whether CBT can be applied with people who have a history of violence and persistent psychotic symptoms and whether this has an impact on reducing violence and aggression. This randomised controlled trial aimed to evaluate the effectiveness of a modified CBT for psychosis programme on outcomes for people with psychosis who had a history of violence (trial registration: NRR NO50087441).

\section{Method}

This was a single-blind randomised controlled trial aimed at comparing CBT in addition to treatment as usual (TAU) compared with a control treatment of social activity therapy (SAT) plus TAU at reducing violence, aggression, anger, psychotic symptoms and risk, and improving functioning in people with schizophrenia. The primary hypothesis was that CBT would be superior in reducing incidents of aggression and violence. Secondary hypotheses were that CBT would be superior in reducing anger, increasing functioning and in reducing psychotic symptoms and risk.

\section{Participants}

Participants were in-patients and out-patients, recruited from mental health services from five National Health Service trusts in the North West of England between 2000 and 2004. Potential participants were identified from mental health staff and from case-note search. Case notes were screened to see whether potential participants met initial inclusion criteria (see below for further details) and those meeting the criteria were interviewed using the Positive and Negative Syndrome Scale ${ }^{7}$ (PANSS) to ascertain whether psychotic symptoms were present. Those who met all criteria were asked whether they would be willing to take part in the trial and those giving consent were subject to the full assessment described below. The inclusion criteria were as follows.

(a) Diagnosis of DSM-IV ${ }^{8}$ schizophrenia or schizoaffective disorder ascertained from multiple sources and case-note checklist. $^{9}$

(b) History of violent behaviour defined as follows: either

(i) at least two recorded incidents of violence to property or others in 12 months prior to trial entry, or

(ii) at least three recorded incidents of verbal aggression threatening violence to property or others in 12 months prior to trial entry, or 
(iii) for participants in secure care, one recorded violent index offence plus an indication that the individual was at risk of further violence without the protective effect of the treatment setting. This was rated by the responsible medical officer or care coordinator using the Security, Dependency, Treatment and Political Needs Assessment Scale. ${ }^{10}$

(c) Experiencing persistent hallucinations and/or delusions scoring four or more on the relevant PANSS sub-scales (P1 and P3).

(d) Receiving antipsychotic medication (dose between $400 \mathrm{mg}$ and $1000 \mathrm{mg}$ chlorpromazine or equivalent) ascertained from detailed case-note review.

Eligible, consenting potential participants were assessed by masked raters at baseline, after 6 weeks (baseline 2), at end of treatment (6 months) and at follow-up (12 months). Masking was maintained by ensuring therapists and assessors were housed in separate accommodation, therapy files were kept separately from data and clinical staff was repeatedly instructed not to disclose any knowledge of therapy group to assessors.

\section{Primary outcome: assessment of aggression and violence}

Data on acts of aggression and violence were collected at four time points from case notes by masked assessors:

(a) from 3 months prior to inclusion in trial to baseline 1

(b) from baseline 1 to baseline 2 (6 weeks)

(c) from baseline 2 to the end of treatment (6 months)

(d) from end of treatment to follow-up (6 months).

Data were collected on eight aggression and violence variables taken from the Ward Anger Rating Scale (WARS; see below, details available from R.W.N. on request) and were recorded if a description of an event was written in any part of the case notes during the target period. From these data, the following three variables were computed: total number of any incidents across each of the four time points by group; number of verbal incidents across each time period by group; and the number of acts of physical violence carried out over the four time periods by group. In addition, a dichotomous variable of 'no violence' v. 'presence of violence' over the intervention and follow-up period was recorded for each individual.

\section{Secondary outcomes}

\section{Staff-rated aggression and anger}

The Ward Anger Rating Scale (WARS) (details available from R.W.N. on request) was used; this is a two-part scale (A and B) completed by a staff member who has observed participants' behaviour during the previous week. Part A consists of 18 dichotomous, weekly ratings regarding verbal and physical behaviours associated with anger and aggression. Eight of the Part A items relate to verbal and physical aggression to property or others (e.g. verbally abused someone, verbally threatened to attack staff member). These were summed to form an index of 'angry-aggressive behaviours'. Part B consists of seven items regarding affective-behavioural attributes related to anger (e.g. angry or annoyed, irritable or grouchy) that staff rate on a fivepoint scale (0-4). The sum of the seven Part B anger attribute ratings produce an 'anger attributes index'.

Good interrater reliability, internal consistency and validity have been demonstrated for the WARS in forensic and in-patient populations. ${ }^{11,12}$

\section{Assessment of self-reported anger}

The Novaco Anger Scale and Provocation Inventory ${ }^{13}$ (NAS-PI) was used: it is a self-report instrument containing cognitive, arousal and behavioural sub-scales, which comprise a total score. The sub-scales relate to the three dispositional domains described by Novaco. ${ }^{14}$ It has received independent validation ${ }^{15}$ and has shown good internal reliability and test-retest reliability. ${ }^{13,14}$ The Provocation Inventory is an anger reaction inventory that was developed to accompany the NAS. It has 25 items providing an index of anger intensity and generality across a range of potentially provocative situations. The Provocation Inventory has been independently validated and has good psychometric properties. $^{13}$

\section{Symptom assessment}

The PANSS ${ }^{7}$ was used to rate severity of psychotic symptoms. Assessors were trained to a high standard of interrater reliability (PANSS positive sub-scale inter-class correlation $=0.905,95 \%$ CI 0.753-0.980).

The Psychotic Symptom Rating Scales (PSYRATS) ${ }^{16}$ was also used to assess symptoms. This uses semi-structured interviews that assess dimensions of psychotic symptoms. They comprise two scales that rate auditory hallucinations and delusions. The scales have been used widely to assess these symptoms and have good psychometric properties with individuals with chronic, and acute, psychosis. ${ }^{17}$ Assessment of functioning was carried out using the Global Assessment of Functioning scale (GAF). ${ }^{8}$

\section{Assessment of risk}

The Historical, Clinical, Risk Management-20 (HCR-20) scale ${ }^{18}$ was used to assess risk; this 20 -item scale consists of three subscales relating to risk of violence. The three sub-scales are: historical factors; clinical factors; and risk factors in relation to the future. Each scale is scored separately by raters who are informed from multiple sources. The scale has been widely evaluated with in-patient, community and forensic groups and has been shown to have good interrater reliability and predictive validity in relation to future violence. The historical scale was only carried out at baseline and the clinical and risk scales were carried out at baseline and follow-up only.

Participant risk status was assessed during the intervention and follow-up period from data collected from multiple sources. Any change in risk management was recorded by trial staff during the whole of the study period. This was also verified from casenote records at the end of the trial by research assistants. For example, changes in amount of freedom within the ward environment, changes in access to sharp objects, increase in access to hospital grounds. The nature of the change in leave status was recorded and, retrospectively, each individual was categorised as either receiving no change or increase in the risk management, or decrease in the risk management.

\section{Treatment}

Participants were randomly allocated to group by personnel independent of the trial using a computer-generated randomisation procedure stratified for gender, presence of substance misuse, severity of anger, presence or absence of actual violence in the last 12 months and facility (out-patient $v$. in-patient). Participants were allocated to either:

(a) a CBT programme which included motivational strategies to aid engagement, CBT strategies to reduce the severity and 
distress of psychotic symptoms and CBT strategies to reduce the severity of anger linked to aggression and violence; or

(b) a SAT programme aimed at helping participants to identify activities they enjoyed and helping them to carry these out.

The rationale for the integrated CBT was based on two main factors. First, it was envisaged that this group of individuals might show considerable problems in engaging with therapy and hence the therapy was enhanced with motivational interviewing strategies to improve the potential for engagement in CBT. Second, it has been demonstrated that both psychotic symptoms and anger are implicated in the occurrence of violence, hence strategies to reduce the severity of both were included. The SAT treatment was designed to control for non-specific elements of therapy such as having a one-to-one relationship with an empathic therapist. Both treatments consisted of 25 sessions carried out by therapists who had been trained in the protocol and received ongoing supervision. Therapists who carried out the CBT programme met the British Association of Behavioural and Cognitive Psychotherapies' Minimum Training Standards for the practice of CBT and had prior experience of applying CBT for people with psychosis. Stringent procedures to manage treatment fidelity were employed as follows.

(a) A therapy manual for each treatment was developed during

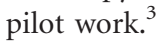

(b) Individual and group supervision was provided at least fortnightly.

(c) Where possible, sessions were tape-recorded and samples of these were listened to by supervisors to ensure therapists were carrying out the treatment according to the protocol. Tapes from the CBT treatment were also assessed by supervisors using the Cognitive Therapy Scale for Psychosis ${ }^{19}$ to ensure therapists were carrying out treatment to a high standard and met criteria for CBT. Similarly, SAT tapes were also rated on the same scale to ensure that the therapists were not carrying out CBT techniques and to ensure that the SAT met non-specific therapeutic quality standards which are measured on the scale (e.g. understanding, empathy, feedback).

(d) An independently randomly selected sample of 20 audio-tapes of intervention sessions (10 CBT, 10 SAT) from the pool of 264 available therapy tapes were assessed by a clinical psychologist independent of the trial and masked to the therapy status of the tapes in order to identify the nature of the treatment being carried out (i.e. either CBT or SAT). All tapes were accurately identified. Treatment was carried out during a 6-month therapy envelope. Participants were assessed by masked researchers at baseline 1 , baseline 2, 6- and 12month follow-up and were assessed on the outcomes described above.

\section{Statistical analysis}

Data were analysed using SPSS for Windows version 14. All analyses were conducted on an intention-to-treat basis. Chisquared analysis was used to assess differences between groups on occurrence of violence and change in risk management. Non-parametric analysis was carried out on violence frequency data and staff-rated anger. Group differences on other quantitative outcomes were examined in a repeated measures analysis using the SPSS mixed model REML procedure (SPSS version 12), contrasting baseline and baseline 2 scores with end of treatment (time 1) and follow-up (time 2) scores with secondary outcome variables of symptoms, functioning and anger. The significance tests of interest were for the end of treatment (time 1) $\times$ treatment group and follow-up (time 2$) \times$ treatment group interactions. Where scores from assessment measures deviated significantly from a normal distribution, log transformed scores were attempted and where distributions remained skewed or there was significant kurtosis, non-parametric statistics were employed.

\section{Results}

We screened 325 people, 108 of whom were identified as meeting initial inclusion criteria. Thirty-one refused to be assessed to determine eligibility. In all, 77 people were eligible and consented to take part and were allocated to a treatment group. Three participants refused to take part in therapy. In addition, one participant died and one participant moved from the area. Figure 1 shows progression through the trial.

Participants were recruited from out-patient and in-patient settings (19 out-patients, 58 in-patients). A breakdown of the location where participants resided at the time of recruitment is shown in Table 1 together with demographic details.

\section{Therapy participation}

Of the 77 participants, 3 decided not to take part in therapy (2 CBT, 1 SAT) and 1 was transferred to a facility out of region making visits not possible. One person died of natural causes before the end of treatment and is not included further in the analysis. Of the rest, the mean number of sessions attended was 17.0 (s.d.=6.8) sessions for CBT and 17.4 (s.d.=6.6) sessions for SAT. The mean number of minutes of therapy received was 787.7 (s.d.=356.2) for CBT and 894.3 (s.d.=413.3) for SAT. There were

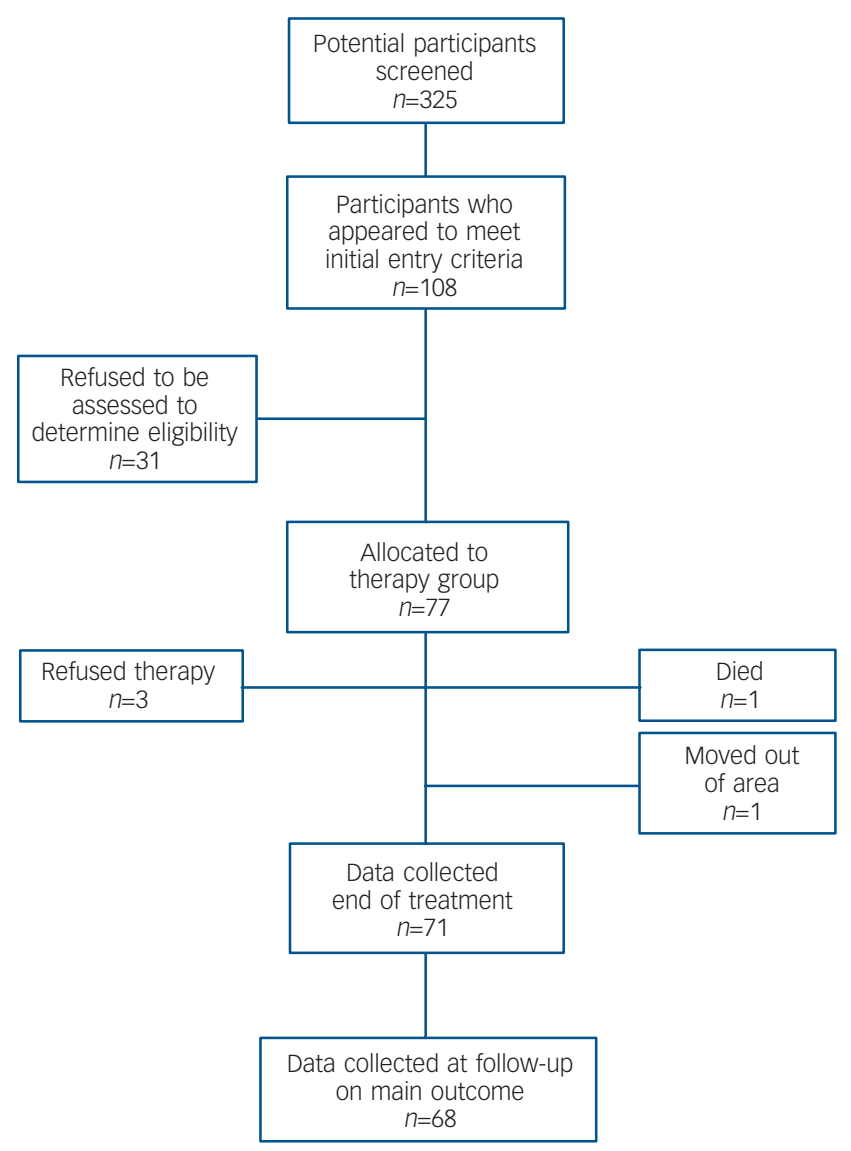

Fig. 1 Progression through the trial. 


\begin{tabular}{|c|c|c|}
\hline & $n$ & $\%$ \\
\hline \multicolumn{3}{|l|}{ Gender } \\
\hline Male & 66 & 85.7 \\
\hline Female & 11 & 14.3 \\
\hline \multicolumn{3}{|l|}{ Diagnosis } \\
\hline Schizophrenia & 69 & 89.6 \\
\hline Schizoaffective disorder & 7 & 9.1 \\
\hline Psychotic illness-NOS & 1 & 1.3 \\
\hline \multicolumn{3}{|l|}{ Marital status } \\
\hline Single & 66 & 85.7 \\
\hline Married & 3 & 3.9 \\
\hline Separated & 8 & 10.4 \\
\hline \multicolumn{3}{|l|}{ Ethnic group } \\
\hline White & 64 & 83.1 \\
\hline Black Caribbean & 3 & 3.9 \\
\hline Black African & 1 & 1.3 \\
\hline Black Other & 2 & 2.6 \\
\hline Indian & 1 & 1.3 \\
\hline Pakistani & 4 & 5.2 \\
\hline Other & 2 & 2.6 \\
\hline \multicolumn{3}{|l|}{ Location of residence } \\
\hline Own home & 4 & 5.2 \\
\hline Supported accommodation & 1 & 1.3 \\
\hline Family home (parents) & 3 & 3.9 \\
\hline Family home (spouse) & 2 & 2.6 \\
\hline Hostel & 9 & 11.7 \\
\hline Ward (not secure) & 10 & 13.0 \\
\hline Ward (secure) & 48 & 62.3 \\
\hline
\end{tabular}

no significant differences between the groups on the number of sessions $(F(1,75)=0.89, P=0.77)$ or minutes of therapy received $(F(1,75)=1.45, P=0.23)$. Of the 73 participants who took part in therapy, all but 8 received at least 10 sessions of therapy. The eight (four from CBT, four from SAT) received between three and nine sessions. Overall, 38 people received CBT and 39 received SAT. There was little difference between the groups in terms of the age of the participants (mean age: $\mathrm{CBT}=35.7$ years, s.d. $=12.5$; SAT $=33.9$ years, s.d. $=9.7$ ).

\section{Primary outcome: case-note identification of aggression and violence}

There was little difference between the treatment groups pre-trial entry ( 3 months prior to baseline 1 ) or between baseline 1 and 2 (pre-treatment intervention) on numbers of people who were aggressive or violent or on total incidents of aggression and violence.

Overall over the treatment period, there were no significant differences between the groups on the number of people who had no incidents of violence or aggression $v$. the number who had one or more incidents $\left(\chi^{2}=2.351\right.$, d.f. $\left.=1, P=0.125\right)$. However, the CBT group had a significantly lower number of incidents during the treatment period $(P=561.0, P=0.039)$. Over the follow-up period, there was no significant difference between the number of people in each group that carried out aggressive or violent acts $\left(\chi^{2}=0.160\right.$, d.f. $\left.=1, P=0.689\right)$ or on total number of incidents (Mann-Whitney $U=697.0, P=0.594$ ). The total number of incidents of verbal or physical aggression recorded from case notes over the intervention and follow-up period by group is shown in Table 2.

There were no significant differences between the groups over the treatment period on the numbers of people who engaged in verbally aggressive acts $\left(\chi^{2}=1.597\right.$, d.f. $\left.=1, P=0.256\right)$ or on the
Table 2 Total number of incidents for each group by case

note identification over the treatment and follow-up period

Incidents, $n$ CBT $(n=38) \quad$ SAT $(n=39)$

During treatment

Verbal aggression

Physical aggression

31

Follow-up

Verbal aggression

Physical aggression

31

2

103

46

CBT, cognitive-behavioural therapy: SAT, social activity therapy.

number of incidents of verbal aggression $(U=611.0, P=0.150)$. This was similar for the follow-up period on number of people $\left(\chi^{2}=0.687\right.$, d.f. $\left.=1, P=0.490\right)$ and number of incidents $(U=555.5$, $P=0.765)$.

Over the treatment period, there were no significant differences between the groups on the number of people who were physically aggressive $\left(\chi^{2}=2.160\right.$, d.f. $\left.=1, P=0.142\right)$ or the total number of incidents involving physical aggression $(U=653.5$, $P=0.172$ ). However, over the follow-up period, there were significantly more people who had been physically aggressive in the SAT group compared with the CBT group $\left(\chi^{2}=8.081\right.$, d.f. $\left.=1, P=0.004\right)$ and there was a significantly lower number of physically aggressive incidents in the CBT group ( $U=466.0, P=0.028$ ).

\section{Secondary outcomes}

Staff-rated anger and aggression

The number of participants scoring positive for aggressive behaviours on the WARS-A scale as rated by staff over a 1-week time period did not differ significantly between the two groups (CBT $n=7$ (end of treatment) and 6 (at follow-up); SAT $n=12$ (end of treatment) and 7 (follow-up)).

Scores on the WARS-B (the anger indices outcome) indicated a decrease overall at end of treatment and follow-up on the amount staff thought the participants were displaying anger. However, there were no significant time 1 or time $2 \times$ group interactions and we conclude that there are no differences between the two treatments on this outcome. Raw scores at each time point can be found in the online Table DS1.

\section{Anger}

No significant time 1 or time $2 \times$ group interactions were found on NAS total anger scores, on any sub-scales or on the Provocation Inventory. The NAS and Provocation Inventory raw scores are shown in Table DS1.

\section{Symptom outcomes}

The PANSS, PSYRATS and GAF raw scores $\times$ treatment group at the four time points are shown in Table DS1. There is some sign of improvement in these symptoms over time but there were no significant time $1 \times$ group or time $2 \times$ group interactions, indicating that neither treatment was superior to the other. However, there were significant interactions between group and treatment on total PSYRATS delusions (but not auditory hallucinations) outcomes at end of treatment (but not followup) with those receiving CBT showing greater reductions in the severity of delusions ( $F=9.469,1$ and 75.2 d.f., $P=0.003$ )

\section{Functioning}

Again, there was an improvement in functioning over time in both groups, but there were no time $\times$ group interactions. 
Risk

Change on the HCR-20 was assessed on the clinical and risk scales of the HCR only. Data were collected at baseline 1 and follow-up. There is little evidence of any treatment group differences.

There were significant differences between the groups regarding security risk (in-patients only, $n=48$ ), with the SAT group having more participants who were in the 'no change/increase risk management' category than those who received CBT, and those receiving CBT having more participants who decreased the amount of risk management required $\left(\chi^{2}=6.19(1), P=0.014\right)$.

\section{Discussion}

Aggression and violence exhibited by people with schizophrenia is of significant clinical and public concern and is a frequent occurrence. $^{20}$ Despite this, very little research has been carried out evaluating psychological interventions to address this. The present study examined outcomes from two psychological treatments, CBT and SAT, delivered over 6 months for people with treatment-resistant schizophrenia who had a history of violence across a range of mental health settings. Cognitive-behavioural therapy was superior to the control treatment on reducing incidents of aggression over the treatment and follow-up period. In addition, although CBT was not superior on general symptom outcomes, it was superior on reducing delusion severity and decreasing risk management. Furthermore, the treatment was acceptable to the majority of individuals; drop out rates from therapy were low and the majority of participants had more than ten sessions of therapy. The high retention rate might be related to the in-patient status of the majority of participants, where attendance at sessions may be more convenient than for people living in the community. Nevertheless, the high adherence to therapy is promising in this group who may generally be difficult to engage in psychological treatment. Despite the benefits from the integration of CBT for psychosis and anger on violence and delusion outcomes, there was no similar benefit on anger. It is possible that further benefits on anger may have been achieved with a longer treatment envelope or booster sessions. Indeed, a 6-month intervention for people who have multiple and complex problems, such as those included in this trial, may have been too short a period in which to expect comprehensive and long-lasting change in all areas targeted.

Despite the benefits, it is unclear what the main mechanism of change was. Benefits were observed for both CBT and SAT on symptoms overall, however, the CBT group had a greater improvement in delusions which may have accounted for the greater impact on reducing physical violence, especially as particular delusions have been consistently linked with increased levels of violence in schizophrenia. ${ }^{4,21,22}$ When the descriptive data were examined, there was a relatively large decrease on the PSYRATS delusions factor of 'distress' ${ }^{16}$ in the CBT group compared with a slight increase in the SAT group. It is possible that CBT provided additional strategies to produce a change, not only in the severity of the delusions, but also in the distress associated with them, a benefit not produced by SAT. Whether this was sufficient to produce the reduction in physical aggression seen in this group is unclear.

\section{Limitations and strengths}

There are a number of limitations that should be considered when interpreting the results from this study. First, the sample size was relatively small which may have limited the power of the study to detect treatment differences. Estimates of power based on a review of previous trials of CBT in schizophrenia recommend at least 70 in each group to show differences between groups. ${ }^{1}$ Similarly, the multiple testing in relation to the secondary outcomes may have increased the likelihood of the positive findings happening as a result of chance, particularly in relation to the positive psychotic symptom outcome. Furthermore, the sample was quite heterogeneous with a mix of in-patients, out-patients and those residing in high-security environments. It is possible that the interventions may have had different impacts in different environments. However, an exploratory analysis of the in-patient participants in the sample on violence, symptoms and anger showed no differences to the pattern of findings found for the whole sample. In addition, this study failed to address issues relating to personality in relation to outcome or in the treatment protocol. This may be necessary in future studies as personality factors may be important predictors of violence for some individuals and may be influential in the degree to which an individual engages with therapy. ${ }^{23}$

Despite these limitations, strengths of the study included:

(a) a robust treatment protocol and careful attention to therapist training issues, supervision and treatment fidelity;

(b) masked, independent assessors of outcome who were trained to a high standard of reliability and validity on the outcome measures;

(c) an intervention that was applied in routine settings by therapists who worked within the institutions;

(d) a follow-up period to examine maintenance of any gains; and

(e) outcomes that focused not just on symptoms but on issues to do with anger, functioning, violence and risk.

These are important outcomes particularly for those people residing in in-patient or secure environments where risk and prediction of future risk are especially pertinent. The positive findings regarding $\mathrm{CBT}$ are promising and suggest optimism for further exploration of psychological treatments for people with psychosis and problems with aggression and violence. However, some further modifications and fine tuning of the treatment may be necessary. In the light of these preliminary findings, further evaluation of the approach is warranted.

Gillian Haddock, Christine Barrowclough, School of Psychological Sciences, University of Manchester, UK; Jennifer J. Shaw, Graham Dunn, School of Community Based Medicine, University of Manchester, UK; Raymond W. Novaco Department of Psychology and Social Behavior, University of California at Irvine, California, USA; Nicholas Tarrier, School of Psychological Sciences, University of Manchester, UK

Correspondence: Gillian Haddock, School of Psychological Sciences, Zochonis Building, University of Manchester, Oxford Road, Manchester M13 9PL, UK. Email: gillian.haddock@manchester.ac.uk

First received 1 May 2007, final revision 23 Jan 2008, accepted 9 May 2008

\section{Acknowledgements}

The trial was funded by the North West Research and Development Fund. The authors would like to thank the following people and organisations: Pennine Care NHS Trust, Bolton Salford and Trafford NHS Trust, Manchester Health and Social Care Trust, Merseycare NHS Trust, Lorna Jellicoe-Jones, Paul Hammersley, Fiona Lobban, Celia Vishnick, Neil Jackson, Kerry Manson and Louise Roper.

\section{References}

1 Jones C, Cormac I, Silveira da Mota Neto Jl, Campbell C. Cognitive behaviour therapy for schizophrenia. Cochrane Database Syst Rev 2007; 4: CD000524.

2 Tarrier $\mathrm{N}$, Wykes $\mathrm{T}$. Is there evidence that cognitive behaviour therapy is an effective treatment for schizophrenia? A cautious or cautionary tale? Behav Res Ther 2004; 42: 1377-401. 
3 Haddock G, Lowens I, Brosnan N, Barrowclough C, Novaco RW. Cognitivebehaviour therapy for inpatients with psychosis and anger problems within low secure environment. Behav Cognit Psychother 2004; 32: 77-98.

4 Appelbaum PS, Robbins PC, Monahan J. Violence and delusions: data from the MacArthur Violence Risk Assessment Study. Arch Gen Psychiatry 2000; 157: $566-72$.

5 Meehan J, Flynn S, Hunt IM, Robinson J, Bickley H, Parsons R, et al. Perpetrators of homicide with schizophrenia: a national clinical survey in England and Wales. Psychiatr Serv 2006; 57: 1648-51.

6 Renwick SJ, Black L, Ramm M, Novaco RW. Anger treatment with forensic hospital patients. Legal Criminol Psychol 1997; 2: 103-16.

7 Kay SR, Opler LA, Lindenmayer JP. The positive and negative syndrome scale (PANSS): rationale and standardisation. Br J Psychiatry 1989; 155 (suppl 7): S59-67.

8 American Psychiatric Association. The Diagnostic and Statistical Manual of Mental Disorders (4th edn) (DSM-IV). APA, 1994.

9 Lewis S, Tarrier N, Haddock G, Bentall RP, Kinderman P, Kingdon D, et al. Randomised controlled trial of cognitive-behavioural therapy in early schizophrenia: acute-phase outcomes. Br J Psychiatry 2002; 181 (suppl 43): s91-s97.

10 Shaw J, Davies J, Morey H. An assessment of the security, dependency and treatment needs of all patients in secure services in a UK health region. J Forensic Psychiatr 2001; 3: 610-37.

11 Taylor JL, DuQueno L, Novaco RW. Piloting a ward anger rating scale for older adults with mental health problems. Behav Cognit Psychother 2004; 32: 1-13.

12 Doyle M, Dolan M. Evaluating the validity of anger regulation problems, interpersonal style, and disturbed mental state for predicting inpatient violence. Behav Sci Law 2006; 24: 783-98.

13 Novaco R. The Novaco Anger Scale and Provocation Inventory. Western Psychological Association, 2003.
14 Novaco RW. Anger as a risk factor for violence amongst the mentally disordered. In Violence and Mental Disorders: Developments in Risk Assessment (eds J Monahan \& HJ Steadman): 21-59. Chicago University Press, 1994

15 Monahan J, Steadman HJ, Silver E, Appelbaum PS, Robbins PC, Mulvey EP, et al. Rethinking Risk Assessment: The MacArthur Study of Mental Disorder and Violence. Oxford University Press, 2001.

16 Haddock G, McCarron J, Tarrier N, Faragher EB. Scales to measure dimensions of hallucinations and delusions: the psychotic symptom rating scales. Psychol Med 1999; 29: 879-89.

17 Drake R, Haddock G, Tarrier N, Bentall R, Lewis S. The Psychotic Symptom Rating Scales (PSYRATS): their usefulness and properties in first episode psychosis. Schizophr Res 2007; 89: 119-22.

18 Webster CD, Douglas KS, Eaves, D, Hart SD. HCR-20: Assessing Risk for Violence (version 2). Simon Fraser University, 1997.

19 Haddock G, Devane S, Bradshaw T, McGovern J, Tarrier N, Kinderman P, et al. An investigation into the psychometric properties of the Cognitive Therapy Scale for Psychosis (CTS-Psy). Behav Cognit Psychother 2001; 29: 221-33.

20 Dean K, Walsh E, Morgan C, Demjaha A, Morgan K, Lloyd T, et al. Aggressive behaviour at first contact with services: findings from the AESOP First Episode Psychosis Study. Psychol Med 2007; 37: 547-57.

21 Bjørkly S. Psychotic symptoms and violence toward others - a literature review of some preliminary findings Part 1. Delusions. Aggression Violent Behav 2002; 7: 617-31.

22 Brennan PA, Mednick SA, Hodgins, S. Major mental disorders and criminal violence in a Danish birth cohort. Arch Gen Psychiatry 2000; 57: 494-500.

23 Hodgkins S, Hiscoke UL, Freese R. The antecedents of aggressive behavior among men with schizophrenia: a prospective investigation of patients in community treatment. Behav Sci Law 2003; 21: 521-46. 